\title{
Sodium-Glucose Co-Transporter 2 Inhibitors Increase Serum Level of Total Procollagen Type 1 Amino-Terminal Propeptide and Bone Strength in Japanese Patients With Type 2 Diabetes Mellitus
}

\author{
Masataka Kusunoki a, g, Naomi Wakazono ${ }^{\text {a }}$, Daisuke Sato ${ }^{b}$, Tetsuro Miyata ${ }^{c}$, , \\ Kazuhiko Tsutsumie, Yoshiharu Oshida ${ }^{\mathrm{f}}$
}

\begin{abstract}
Background: Diabetes mellitus is known to be associated with an increased risk of bone fracture. We investigated the effects of sodiumglucose co-transporter 2 (SGLT2) inhibitors on bone strength and density in type 2 diabetic patients.

Methods: Nineteen Japanese patients with type 2 diabetes mellitus were administered $2.5 \mathrm{mg}$ /day of luseogliflozin or $5 \mathrm{mg}$ /day of dapagliflozin for 6 months. Serum levels of tartrate-resistant acid phosphatase 5b (TRACP-5b), an osteoclastic marker, and total procollagen type 1 amino-terminal propeptide (P1NP), a bone formation marker, were measured and compared with those before the treatment. Bone strength was measured by quantitative ultrasound (QUS), and bone density was evaluated by dual-energy X-ray absorptiometry (DEXA).
\end{abstract}

Results: SGLT2 inhibitors significantly increased the calcaneal bone strength as measured by QUS compared to that in young adult mean. However, there was no effect in the lumbar spine density as measured by DEXA after administration. The drug treatment had no effect on serum TRACP-5b, but significantly increased serum P1NP.

Manuscript submitted August 6, 2020, accepted August 13, 2020

Published online August 26, 2020

${ }^{a}$ Research Center of Health, Physical Fitness and Sports, Nagoya University, Furo-cho, Chikusa-ku, Nagoya 464-8601, Japan

${ }^{b}$ Department of Biochemical Engineering, Graduate School of Science and Engineering, Yamagata University, 4-3-16 Johnan, Yonezawa 992-8510, Japan 'Office of Medical Education, School of Medicine, International University of Health and Welfare, 4-3 Kozunomori, Narita 286-8686, Japan

dVascular Center, Sanno Medical Center, 8-5-35 Akasaka, Minato-ku, Tokyo 107-0052, Japan

'Okinaka Memorial Institute for Medical Research, 2-2-2 Toranomon, Minato-ku, Tokyo 105-8470, Japan

${ }^{f}$ Medical Checkup Center, Minami Seikyo Hospital, 2-204 Minami-odaka, Midori-ku, Nagoya 459-8540, Japan

'Corresponding Author: Masataka Kusunoki, Research Center of Health, Physical Fitness and Sports, Nagoya University, Furo-cho, Chikusa-ku, Nagoya 464-8601, Japan. Email: info@tonyo.jp

doi: https://doi.org/10.14740/jem683
Conclusions: The results imply that the SGLT2 inhibitors improve bone strength while the inhibitors had no effect on bone density. These results suggest that the increase in bone strength was due to improved bone quality through an increase in serum P1NP level.

Keywords: SGLT2 inhibitor; Bone fracture; Quantitative ultrasound analysis; Dual-energy X-ray absorptiometry; Procollagen type 1 amino-terminal propeptide

\section{Introduction}

Type 2 diabetic patients are known to be at a high risk for bone fracture [1]. In addition, obesity, particularly visceral obesity, has also been reported to be associated with a low bone mass and an increased risk of fracture [2]. Regarding the effects of antidiabetic agents on bone fractures, canagliflozin, an sodium-glucose co-transporter 2 (SGLT2) inhibitor, has been reported to increase fracture risk in diabetic patients $[3,4]$; and Blau et al [3] reported that increased blood phosphate, intact fibroblast growth factor 23 (FGF23) and parathyroid hormone (PTH) and decreased 1,25-dihydroxyvitamin D are involved in the mechanism behind this effect.

However, there is no consensus on the effects of administration of antidiabetic agents on the risk of fracture or on the bone metabolism in type 2 diabetic patients.

Selective inhibitors of SGLT2 have been reported to exert hypoglycemic effects by selectively inhibiting SGLT2 expressed in the renal proximal tubules, thereby suppressing the reabsorption of glucose filtered by the glomerulus, leading to urinary excretion of the excess blood glucose $[5,6]$. It has also been reported that these inhibitors not only lower the blood glucose, but also decrease the body weight, lower the blood pressure and improve lipid and uric acid metabolism [7]. However, there have been few reports on the effects of SGLT2 inhibitors on bone metabolism.

We previously reported that treatment with three SGLT2 inhibitors (luseogliflozin, dapagliflozin, and tofogliflozin) was associated with improved bone strength as measured by quantitative ultrasound (QUS) in Japanese patients with type 
2 diabetes mellitus [8]. However, we did not simultaneously perform dual-energy X-ray absorptiometry (DEXA), which is used to measure the bone density. In the present study, we newly investigated the effects of treatment with two SGLT2 inhibitors (luseogliflozin and dapagliflozin) on the bone strength and density in Japanese patients with type 2 diabetes mellitus, by performing QUS and DEXA before and after treatment. In addition, blood levels of tartrate-resistant acid phosphatase $5 \mathrm{~b}$ (TRACP-5b), which is an osteoclastic marker, and total procollagen type 1 amino-terminal propeptide (P1NP), which is a bone formation marker, were measured, to investigate the effects of the drug treatment on bone metabolism.

\section{Materials and Methods}

\section{Study design}

The present study was conducted in compliance with the ethical standards of the responsible institution on human subjects as well as with the Helsinki Declaration. The present study, which was conducted at Akishina Clinic (Nagoya, Japan), was approved by the institutional ethics committee (approval number: 1-1). Patients who participated in the study were explained the purpose of the study by the physicians in charge, and gave informed consent. This clinical study is officially registered as an open-label study (ID: UMIN000021584).

The study subjects were 19 patients with type 2 diabetes mellitus (16 males and three females), who were attending the outpatient clinic and gave consent for participation in the clinical study. The subjects included patients who were receiving antidiabetic, lipid-lowering or antihypertensive agents other than SGLT2 inhibitors. Patients receiving drug treatment for osteoporosis or estrogen replacement therapy were not included in this study.

The subjects received $2.5 \mathrm{mg}$ of luseogliflozin $(\mathrm{n}=13)$ or $5 \mathrm{mg}$ of dapagliflozin $(\mathrm{n}=6)$ once daily before or after breakfast for 6 months. Bone strength was measured by QUS analysis, and bone density was measured by DEXA before and after 6 months' administration. The bone strength and density were expressed as comparison with those in young adult means.

In addition, to measure the biochemical parameters, blood samples were collected before and after 6 months of treatment, serum was separated, and the serum samples were stored frozen until the measurements.

Achilles Express A-1000 (GE Healthcare UK Ltd, England) was used for QUS analysis and Dichroma Scan DCS900FX (Hitachi Healthcare Business Service, Ltd, Japan) for DEXA.

\section{Blood analyses}

Measurement of the hemoglobin A1c (HbA1c), serum levels of total cholesterol (TC), high-density lipoprotein cholesterol (HDL-C), low-density lipoprotein cholesterol (LDL-C), triglyceride (TG), TRACP-5b, and total P1NP were entrusted to Handa Medical Association Health Center (Aichi, Japan);
Table 1. Characteristics of Patients Enrolled in the Study

\begin{tabular}{|c|c|}
\hline Number of patients & 19 \\
\hline Male/female & $16 / 3$ \\
\hline Age (years) & $66 \pm 12$ \\
\hline Body weight(kg) & $73.7 \pm 10.9$ \\
\hline $\operatorname{BMI}\left(\mathrm{kg} / \mathrm{m}^{2}\right)$ & $26.6 \pm 3.2$ \\
\hline HbAlc (\%) & $7.4 \pm 1.1$ \\
\hline \multicolumn{2}{|l|}{ Concomitant medication } \\
\hline \multicolumn{2}{|l|}{ Anti-diabetic drug (n) } \\
\hline Biguanide & 6 \\
\hline Sulfonylurea & 1 \\
\hline Insulin & 1 \\
\hline Alpha-glucosidase inhibitor & 5 \\
\hline Thiazolidinedione & 3 \\
\hline DPP4-inhibitor & 10 \\
\hline Lipid-modifying agents (n) & 13 \\
\hline Antihypertensive drug (n) & 13 \\
\hline
\end{tabular}

Data are expressed as mean \pm SD. SD: standard deviation; BMI: body mass index; HbA1c: hemoglobin A1c; DPP4: dipeptidyl peptidase 4.

serum lipids were measured using an auto-analyzer (JCABM8000 series, JAOL, Tokyo, Japan), and HbAlc level was measured by automated high-performance liquid chromatography (HLC-723GX, Tosoh Corporation, Tokyo, Japan). TRACP-5b was measured by enzyme immunoassay and total P1NP was measured by electrochemiluminescence immunoassay.

\section{Statistical analysis}

Data are shown as means \pm standard deviation (SD). Values measured before and after 6 months' treatment were compared by the paired $t$-test, with the significance level set at $\mathrm{P}<0.05$.

\section{Results}

\section{Patient characteristics}

The patient characteristics are shown in Table 1, including the antidiabetic, lipid-lowering and antihypertensive agents that the patients were receiving in addition to the SGLT2 inhibitors.

\section{Effects of SGLT2 inhibitors on body mass index (BMI), HbA1c, and serum glucose and lipids}

The results of treatment with either of the two SGLT2 inhibitors for 6 months are shown in Table 2. The SGLT2 inhibitor treatment for 6 months was associated with a significant decrease of the body weight and BMI as compared to the values recorded 
Table 2. Effects of Six Months' Administration of SGLT2 Inhibitors on the Body Weight, BMI, HbA1c, and Glucose- and LipidMetabolic Parameters

\begin{tabular}{lll} 
& Before administration & After $\mathbf{6}$ months' administration \\
\hline Body weight $(\mathrm{kg})$ & $73.7 \pm 10.9$ & $70.6 \pm 11.2^{* *}$ \\
BMI $\left(\mathrm{kg} / \mathrm{m}^{2}\right)$ & $26.6 \pm 3.2$ & $25.5 \pm 3.2^{* *}$ \\
HbAlc $(\%)$ & $7.4 \pm 1.1$ & $6.8 \pm 0.8^{*}$ \\
Blood glucose $(\mathrm{mg} / \mathrm{dL})$ & $115 \pm 36$ & $98 \pm 17^{*}$ \\
Serum total cholesterol $(\mathrm{mg} / \mathrm{dL})$ & $190 \pm 30$ & $187 \pm 23$ \\
Serum LDL-cholesterol (mg/dL) & $112 \pm 26$ & $111 \pm 20$ \\
Serum HDL-cholesterol (mg/dL) & $52 \pm 10$ & $59 \pm 9^{* *}$ \\
Serum triglyceride $(\mathrm{mg} / \mathrm{dL})$ & $186 \pm 94$ & $117 \pm 67^{* *}$ \\
\hline
\end{tabular}

Data are expressed as mean $\pm \mathrm{SD}$. ${ }^{*} \mathrm{P}<0.05$, ${ }^{*} \mathrm{P}<0.01$ vs. before administration. SD: standard deviation; BMI: body mass index; HbA1c: hemoglobin A1c; SGLT2: sodium-glucose co-transporter 2; LDL: low-density lipoprotein; HDL: high-density lipoprotein.

prior to the start of treatment administration $(\mathrm{P}<0.01)$.

In addition, the blood $\mathrm{HbA} 1 \mathrm{c}$ and glucose levels also decreased significantly $(\mathrm{P}<0.05)$. In regard to the effects on the blood lipid levels, the SGLT2 inhibitor treatment significantly increased the serum level of HDL-C $(\mathrm{P}<0.01)$ and significantly decreased the serum levels of TG $(\mathrm{P}<0.01)$; however, it had no effect on the serum levels of TC or LDL-C.

\section{Effects of SGLT2 inhibitors on bone metabolism param- eters, strength, and density}

The results of treatment with either of the two SGLT2 inhibitors on the bone strength, bone density and bone metabolism parameters are shown in Table 3.

Evaluation of the effects on the bone strength by QUS revealed that the SGLT2 inhibitor treatment was associated with a significant increase of the calcaneal bone strength ( $\mathrm{P}$ $<0.05)$. Assessment of bone density by DEXA revealed that the SGLT2 inhibitor treatment had no effect on the lumbar or femoral bone density.

Blood levels of total P1NP, a bone formation marker, were measured before and after the SGLT2 inhibitor treatment. The levels were found to be significantly higher after the treat- ment as compared to that prior to the treatment $(\mathrm{P}<0.05)$. The SGLT2 inhibitor treatment had no effect on the blood levels of TRACP-5b, a marker of osteoclastic activity.

\section{Discussion}

Diabetes mellitus are associated with a high fracture risk [1]. An approximately 1.4-fold increase in the risk of femoral fracture in patients with type 2 diabetes mellitus as compared to the risk in non-diabetic individuals has been reported [9]. In addition, a 4.7-fold increase in vertebral fracture risk in male type 2 diabetic patients and a corresponding increase in the risk by 1.9 -fold in female type 2 diabetic patients, as compared with the risk in non-diabetic patients, have been reported [10]. In regard to the cause of the increased fracture risk in patients with diabetes mellitus, a study using a rat model of diabetes mellitus has suggested that the enhancement of oxidative stress due to hyperglycemia causes an increase in the release of advanced glycation end products (AGEs) in the bone, leading to deterioration of the bone quality [11]. For reducing the fracture risk, it is considered important to lower the blood glucose and thereby suppress increase in the blood levels of AGEs.

A study of the effects of insulin on the fracture risk by

Table 3. Effects of Six Months' Administration of SGLT2 Inhibitors on Bone Strength, Bone Density and Bone Metabolism-Related Parameters

\begin{tabular}{|lll|}
\hline & Before administration & After 6 months' administration \\
\hline $\begin{array}{l}\text { Bone strength measured by QUS } \\
\quad \text { Calcaneal bone }(\%)\end{array}$ & $88 \pm 16$ & $91 \pm 15^{*}$ \\
\hline $\begin{array}{l}\text { Bone density measured by DEXA } \\
\text { Lumbar spine (\%) }\end{array}$ & $109 \pm 13$ & $106 \pm 12$ \\
$\quad$ Femur $(\%)$ & $87 \pm 13$ & $83 \pm 10$ \\
Serum total P1NP $(\mu \mathrm{g} / \mathrm{L})$ & $35.2 \pm 10.3$ & $39.0 \pm 12.8^{*}$ \\
\hline Serum TRACP-5b $(\mathrm{mU} / \mathrm{dL})$ & $215 \pm 62$ & $245 \pm 82$ \\
\hline
\end{tabular}

Data are expressed as mean $\pm S D$. ${ }^{*} P<0.05$ vs. before administration. Bone strength and density are expressed as the comparison with those in young adult means. QUS: quantitative ultrasound; DEXA: dual-energy X-ray absorptiometry; SD: standard deviation; SGLT2: sodium-glucose cotransporter 2 . 
Schneider et al reported that the fracture risk is higher in longstanding diabetic patients with $\mathrm{HbA} 1 \mathrm{c} \geq 7.5 \%$, who are receiving insulin treatment [12]. In addition, a 1.5- to 2.5-fold higher risk of fracture has been reported in female diabetic patients receiving treatment with thiazolidine derivatives [13]. Furthermore, Vestergaard et al reported that the fracture risk decreased by 0.81 -fold in diabetic patients who were receiving metformin [14]. Treatment with DPP-4 inhibitors has also been shown to reduce the fracture risk by $40 \%$ as compared to that in untreated controls [15].

The SGLT2 inhibitor canagliflozin was reported to increase fracture risk $[3,4]$. However, the mechanism by which canagliflozin increases fracture risk has not been fully understood. Blau et al reported that increased blood phosphate, intact FGF23 and PTH and decreased 1,25-dihydroxyvitamin D were involved in the mechanism [3]. In addition, canagliflozin was reported to increase collagen type $1 \beta$-carboxy-telopeptide levels and bone resorption, resulting in an increased risk of bone fracture [16].

In the present study, we treated Japanese type 2 diabetic patients with one of two SGLT2 inhibitors (luseogliflozin and dapagliflozin) for 6 months and investigated the effects of the treatment on the fracture risk and bone metabolism markers. As part of the evaluation of the treatment effects on the fracture risk, we performed QUS to measure the bone strength [17] and DEXA to measure the bone density [18]. QUS is a convenient and useful measurement method for bone strength, which gives no X-ray exposure and can measure many patients.

This study showed that treatment with either of the two SGLT2 inhibitors, like previously reported SGLT2 inhibitors, was associated with a decrease of the body weight and BMI, and lower blood glucose and HbAlc levels. In addition, these SGLT2 inhibitors also lowered the blood levels of triglycerides and increased those of HDL-C.

In regard to the effects of the treatment on the fracture risk, we previously reported that treatment with one of three SGLT2 inhibitors (luseogliflozin, dapagliflozin and tofogliflozin) improved the calcaneal bone strength as measured by QUS in Japanese type 2 diabetic patients [8], while we did not perform DEXA, which is used to measure the bone density. In the present study, to investigate the effects of treatment with one of the two SGLT2 inhibitors (luseogliflozin and dapagliflozin) on the bone strength and density, we employed two evaluation methods, namely, QUS and DEXA. Similar to our preceding study, the present study also confirmed that treatment with SGLT2 inhibitors increases the calcaneal bone strength as measured by QUS. On the other hand, DEXA, which is used to measure the bone density, revealed no effect of SGLT2 inhibitor treatment on the bone density. Bone strength is thought to be the sum of bone density and bone quality, and in the present study, an increase of the bone strength following SGLT2 inhibitor treatment with no change of the bone density suggested an improvement of the bone quality as underlying the increase in the bone strength.

In regard to the effects of SGLT2 inhibitor treatment on the bone metabolism markers, the treatment induced no change in the blood levels of TRACP-5b, an osteoclastic marker [19]. On the other hand, the SGLT2 treatment significantly increased the blood total levels of the bone formation marker, P1NP $(\mathrm{P}<0.05)[20]$.
Thus, the results of this study revealed that the increased bone strength observed after treatment with either of the two SGLT2 inhibitors was associated with an improved bone quality, mediated by an increase in the blood levels of the bone formation marker, P1NP. However, the mechanism by which the SGLT2 inhibitors increased the blood level of total P1NP remains unknown. In the future, it is necessary to elucidate the mechanism by which SGLT2 inhibitors increase the blood levels of total P1NP. In addition, this study was conducted in only 19 patients, and we believe that large-scale clinical studies are necessary. We plan to measure FGF23, osteocalcin, serum and urine calcium, PTH, and 1,25-dihydroxyvitamin D in such future clinical studies.

In this study, the correlation between bone strength and $\mathrm{HbA} 1 \mathrm{c}$ and also the correlation between bone strength and body weight before and after treatment were examined. The results indicated the rate of change of $\mathrm{HbAlc}$ was not correlated with the rate of change of bone strength $(\mathrm{r}=-0.269, \mathrm{P}=$ 0.11 ), and also the rate of change of body weight was not correlated with the rate of change of bone strength $(\mathrm{r}=0.382, \mathrm{P}$ $=0.27$ ). This suggests that improvement of bone strength may not relate to reductions in $\mathrm{HbA} 1 \mathrm{c}$ and body weight.

The limitations of this study were the small number of female patients and necessity of further investigation of key parameters (FGF23, PTH, 1,25-dihydroxyvitamin D, AGEs). We plan to conduct further studies.

In conclusion, treatment with either of two SGLT2 inhibitors (luseogliflozin and dapagliflozin) was associated with an increase in the bone strength, with no effect on the bone density, in Japanese type 2 diabetic patients. This is likely because the SGLT2 inhibitors improved the bone quality through inducing increase in the serum total P1NP.

\section{Acknowledgments}

None to declare.

\section{Financial Disclosure}

None to declare.

\section{Conflict of Interest}

All the authors report no conflict of interest.

\section{Informed Consent}

The informed consents were obtained.

\section{Author Contributions}

MK contributed to the conception and interpretation of the data. NW and KT performed the statistical analysis. MK, NW, 
$\mathrm{TM}, \mathrm{KT}$, and $\mathrm{YO}$ were involved in writing, reviewing, and editing the manuscript. All the authors read and approved the final manuscript.

\section{Data Availability}

The authors declare that data supporting the findings of this study are available within the article.

\section{Abbreviations}

AGE: advanced glycation end product; DEXA: dual-energy X-ray absorptiometry; FGF23: fibroblast growth factor 23; HbA1c: hemoglobin A1c; HDL-C: high-density lipoprotein cholesterol; LDL-C: low-density lipoprotein cholesterol; P1NP: procollagen type 1 amino-terminal propeptide; PTH: parathyroid hormone; QUS: quantitative ultrasound; TC: total cholesterol; TG: triglyceride; TRACP-5b: tartrate-resistant acid phosphatase $5 \mathrm{~b}$

\section{References}

1. Schwartz AV, Vittinghoff E, Bauer DC, Hillier TA, Strotmeyer ES, Ensrud KE, Donaldson MG, et al. Association of BMD and FRAX score with risk of fracture in older adults with type 2 diabetes. JAMA. 2011;305(21):21842192.

2. Cohen A, Dempster DW, Recker RR, Lappe JM, Zhou H, Zwahlen A, Muller R, et al. Abdominal fat is associated with lower bone formation and inferior bone quality in healthy premenopausal women: a transiliac bone biopsy study. J Clin Endocrinol Metab. 2013;98(6):2562-2572.

3. Blau JE, Bauman V, Conway EM, Piaggi P, Walter MF, Wright EC, Bernstein S, et al. Canagliflozin triggers the FGF23/1,25-dihydroxyvitamin D/PTH axis in healthy volunteers in a randomized crossover study. JCI Insight. 2018;3(8)

4. Lupsa BC, Inzucchi SE. Use of SGLT2 inhibitors in type 2 diabetes: weighing the risks and benefits. Diabetologia. 2018;61(10):2118-2125.

5. Wright EM, Loo DD, Hirayama BA. Biology of human sodium glucose transporters. Physiol Rev. 2011;91(2):733794.

6. DeFronzo RA, Davidson JA, Del Prato S. The role of the kidneys in glucose homeostasis: a new path towards normalizing glycaemia. Diabetes Obes Metab. 2012;14(1):514.

7. Seino Y, Sasaki T, Fukatsu A, Ubukata M, Sakai S, Samukawa Y. Efficacy and safety of luseogliflozin as monotherapy in Japanese patients with type 2 diabetes mellitus: a randomized, double-blind, placebo-controlled, phase 3 study. Curr Med Res Opin. 2014;30(7):1245-1255.

8. Kusunoki M, Natsume Y, Sato D, Tsutsui H, Sasaki H, Nakamura T, Miyata T, et al. Efficacy of SGLT2 inhibitors on bone mineral density in Japanese patients with type 2 diabetes. Diabetologia. 2015;58(suppl. 1):S369.

9. Vestergaard P. Discrepancies in bone mineral density and fracture risk in patients with type 1 and type 2 diabetesa meta-analysis. Osteoporos Int. 2007;18(4):427-444.

10. Yamamoto M, Yamaguchi T, Yamauchi M, Kaji H, Sugimoto T. Diabetic patients have an increased risk of vertebral fractures independent of BMD or diabetic complications. J Bone Miner Res. 2009;24(4):702-709.

11. Saito M, Fujii K, Mori Y, Marumo K. Role of collagen enzymatic and glycation induced cross-links as a determinant of bone quality in spontaneously diabetic WBN/Kob rats. Osteoporos Int. 2006;17(10):1514-1523.

12. Schneider AL, Williams EK, Brancati FL, Blecker S, Coresh J, Selvin E. Diabetes and risk of fracture-related hospitalization: the Atherosclerosis Risk in Communities Study. Diabetes Care. 2013;36(5):1153-1158.

13. Zhu ZN, Jiang YF, Ding T. Risk of fracture with thiazolidinediones: an updated meta-analysis of randomized clinical trials. Bone. 2014;68:115-123.

14. Vestergaard P, Rejnmark L, Mosekilde L. Relative fracture risk in patients with diabetes mellitus, and the impact of insulin and oral antidiabetic medication on relative fracture risk. Diabetologia. 2005;48(7):1292-1299.

15. Monami M, Dicembrini I, Antenore A, Mannucci E. Dipeptidyl peptidase- 4 inhibitors and bone fractures: a meta-analysis of randomized clinical trials. Diabetes Care. 2011;34(11):2474-2476.

16. Bilezikian JP, Watts NB, Usiskin K, Polidori D, Fung A, Sullivan D, Rosenthal N. Evaluation of bone mineral density and bone biomarkers in patients with type 2 diabetes treated with canagliflozin. J Clin Endocrinol Metab. 2016;101(1):44-51.

17. Langton CM, Palmer SB, Porter RW. The measurement of broadband ultrasonic attenuation in cancellous bone. Eng Med. 1984;13(2):89-91.

18. Leib ES, Lewiecki EM, Binkley N, Hamdy RC, International Society for Clinical D. Official positions of the International Society for Clinical Densitometry. J Clin Densitom. 2004;7(1):1-6.

19. Alatalo SL, Halleen JM, Hentunen TA, Monkkonen J, Vaananen HK. Rapid screening method for osteoclast differentiation in vitro that measures tartrate-resistant acid phosphatase $5 \mathrm{~b}$ activity secreted into the culture medium. Clin Chem. 2000;46(11):1751-1754.

20. Vasikaran S, Cooper C, Eastell R, Griesmacher A, Morris HA, Trenti T, Kanis JA. International Osteoporosis Foundation and International Federation of Clinical Chemistry and Laboratory Medicine position on bone marker standards in osteoporosis. Clin Chem Lab Med. 2011;49(8):1271-1274. 